Conclusions Using Hitales platform to set up our clinical database can extract medical information conveniently, quickly and efficiently with sufficient accuracy. So far, we only simply analysis the clinical features of SLE patients. With joint of biological specimens' library and follow up data, the LUPUS puzzle could be learned more.

\section{IDENTIFICATION OF THE LONG NONCODING RNA ENST00000602652 AS A NOVEL INFLAMMATORY REGULATOR ACTING THROUGH TYPE I INTERFERON PATHWAY IN SYSTEMIC LUPUS ERYTHEMATOSUS}

Z Liao*1. 'Shanghai Institute of Rheumatology, Rheumatology, Shanghai, China

\subsection{6/lupus-2017-000215.291}

Background and aims Long noncoding RNAs (lncRNAs) have recently been identified to be tightly linked to diverse human diseases. Systemic lupus erythematosus is an autoimmune disease and renal involvement is the most frequent complication. Inflammatory cytokines produced by renal mesangial cells (RMCs) play a vital role in lupus nephritis(LN). In the present study we investigated the contribution of the IncRNA Enst00000602652 to the pathogenesis to LN.

Methods The high throughput RNAseq data between LN and healthy control was used to screen for candidate lncRNA. SYBR Green quantitative RT-PCR(RT-qPCR) was used to detect the expression of IncRNA and individual interferonstimulated genes (ISGs). Western blotting and luciferace was used to confirm the regulatory function of lncRNA.

Results LncRNA Enst00000602652 expression was abnormally increased in LN patients and correlated to degree of renal damage. Additionally, Expression of LncRNA Enst00000602652 was induced by stimulation of type I interferon. Silencing Enst00000602652 significantly reduced the expression of a group of chemokines and cytokines, including IFIT1, oas1, etc., which were induced by type I interferon. Furthermore, LncRNA Enst00000602652 affects IFN receptor I and phosphorylation of Jak1 and Stat1.

Conclusions Long noncoding RNA Enst00000602652 is a positive regulator of the IFN signalling pathway in LN. LncRNA Enst00000602652 may contributes to the pathogenesis of LN and provides potentially novel target for therapeutic intervention.

\section{HIGH FREQUENCY OF CIRCULATING TERMINALLY DIFFERENTIATED CD8+ T CELLS CHARACTERISE SYSTEMIC LUPUS ERYTHEMATOSUS PATIENTS WITH RENAL INVOLVEMENT}

${ }^{1} \mathrm{~N}$ Manjarrez Orduno*, 'L Menard, 'J Carman, 'S Suchard, ' $\mathrm{F}$ Casano, 'D Lee, 'S Daouti, ${ }^{1} \mathrm{~S}$ Habte, ${ }^{1} \mathrm{~S}$ Kansal, ${ }^{2} \mathrm{~S}$ Bandyopadhyay, ${ }^{2} \mathrm{Y}$ Hu, ${ }^{3} \mathrm{R}$ Furie, ${ }^{1} \mathrm{~N}$ Steven. ${ }^{1}$ Bristol-Myers Squibb, Translational Biology, Princeton, USA; ${ }^{2}$ Bristol-Myers Squibb, Translational Bioinformatics, Princeton, USA; ${ }^{3}$ NorthWell Health, Division of Rheumatology, Great Neck, USA

\subsection{6/lupus-2017-000215.292}

Background and aims Systemic lupus erythematosus (SLE) is a highly heterogeneous disease with limited therapeutic options, where clinical manifestations are the result of multiple pathological mechanisms. The elucidation of these mechanisms is critical for identifying novel therapeutic targets and agents that are more likely to benefit individual patients. Here we investigated the role that $\mathrm{CD}^{+} \mathrm{T}$ cells play in SLE.
Methods We studied $\mathrm{CD}^{+} \mathrm{T}$ cell differentiation in SLE patients under standard of care $(n=80)$, from two different cohorts. The analyses included phenotyping of $\mathrm{T}$ cell differentiation, intracellular cytokine staining and whole blood gene expression.

Results We identified a subset of lupus patients that have elevated numbers of terminally differentiated $\mathrm{CD}^{+}{ }^{+} \mathrm{T}$ cells, identified as $\mathrm{CCR}^{-} \mathrm{CD} 45 \mathrm{RA}^{\text {int-hi }} \mathrm{CD} 28^{-}$. This increase in terminally differentiated CD ${ }^{+} \mathrm{T}$ cells is accompanied by an increase in perforin and granzyme $\mathrm{B}$ production and correlated with a whole blood gene module of cytotoxic activity $\left(\mathrm{p}<5 \times 10^{-9}\right)$. More importantly, this phenotype was associated with lupus nephritis $(\mathrm{p}<0.02)$.

Conclusions We have identified a lupus endophenotype, characterised by the increase in terminally differentiated $\mathrm{CD} 8^{+} \mathrm{T}$ cells, which correlated with cytotoxic activity and renal manifestations of the disease. These findings suggest that this group of patients may benefit from therapies that block $\mathrm{CD} 8^{+} \mathrm{T}$ cell activation and differentiation.

\section{EFFECT OF HIGH DOSE CHOLECALCIFEROL DURING SLE FLARES IN MIR-146A EXPRESSION, REGULATORY T- CELLS AND IL-17A EXPRESSION}

${ }^{1} \mathrm{~A}$ Marinho*, ${ }^{2} \mathrm{C}$ Carvalho, ${ }^{2} \mathrm{D}$ Boleixa, ${ }^{2} \mathrm{~A}$ Bettencourt, ${ }^{2} \mathrm{~B}$ Martins da Silva, ${ }^{1} \mathrm{C}$ Vasconcelos ${ }^{1}$ Centro Hospitalar Do Porto, Internal Medicine, Porto, Portugal; ${ }^{2}$ ICBAS, Immunogenetics, Porto, Portugal

\subsection{6/lupus-2017-000215.293}

Background and aims A few microRNAs have known gene expression regulatory roles in innate immunity. The miR-146a, seems to be a negative regulator of innate immunity. Interestingly, miR-146a has been reported to be downregulated in PBMCs of SLE patients, being negatively correlated with clinical disease activity and with IFN levels. The ability of vitamin $\mathrm{D}$ to regulate miRs and their emerging relationship have been proposed through several experimental approaches. The aim of this study was to determine the Vitamin $\mathrm{D}$ effect in miR146a expression and in T-Reg and $\mathrm{TCD}^{+}{ }^{+} \mathrm{IL}-17 \mathrm{~A}$ producing cells, in SLE.

Methods An interventional study with 3 weeks follow-up of SLE patients with a high dose vitamin D supplementation (50.000 UI or $100.000 \mathrm{UI} /$ Week) was done. We assessed four female patients who had a SLEDAI $>6$ and at least one BILAG A. At screening, relevant data were compiled: SLEDAI$2 \mathrm{~K}$, BILAG score, concomitant therapy, previous SLE manifestations, 25(OH)D levels, T-Reg/IL-17A ratio and miR-146a expression. At Week 3: 25(OH) D levels, T-Reg/IL-17A ${ }^{+}$ratio, miR-146a expression, SLEDAI $2 \mathrm{~K}$, BILAG and concomitant therapy.

Results No significant difference were found, regarding Vitamin D levels, before and after supplementation. Regarding Tregs/IL-17A ratio before and after supplementation, no benefits, regarding enhancing of T-regs or decrease of Il 17-A producing cells. No significative differences were found in miR146a expression between controls and SLE active patients before and after vitamin D supplementation.

Conclusions Severe SLE activity may cause resistance to Vitamin D therapeutical effects, including enhancing of Vitamin D levels and immunogenetic effects. 